\title{
P. 0905 Complications of Diabetes Mellitus Type 2 in Medical Ward Lahore General Hospital
}

Khan, M.I.H.(1)*;Cheema, J.M.(2)

Department of Medicine, Division of Diabetes, Endocrine and Metabolism

Postgraduate Medical Institute/Ameer ud din Medical College

Lahore General Hospital, Lahore

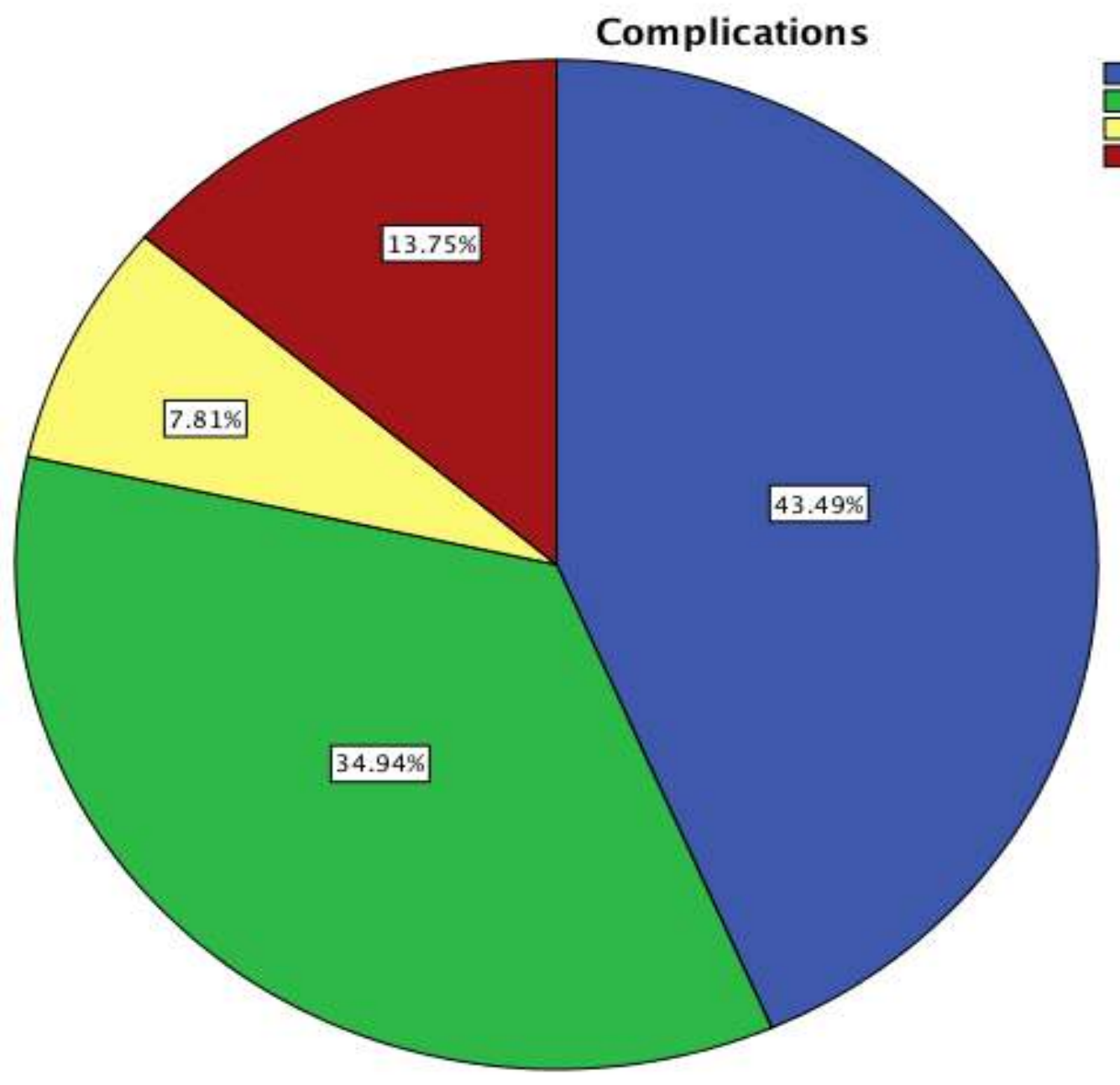

Renal faliure
diabetic foot
Neuropathy
Nil

ABSTRACT

\section{OBJECTIVE:}

This study was conducted at Lahore General Hospital, Punjab to explore the pattern of common complications in Diabetes Mellitus Type 2 patients, their relation to the duration of diabetes and to compare between the genders.

\section{METHOD:}

This is a retrospective study based on patients record involving 269 patients (171 Men and 98 females) who were admitted with diabetic complications in medical ward during a period of 2years (January 2014 to December 2015). Data was analyzed on SPSS 20 and the following results were obtained.

\section{RESULTS:}

Only patients with Type II DM were included in this study. 232 patients (86.2\%) out of 269 had one or more diabetic complications. In general, the most common diabetic complication was diabetic nephropathy 117 (43.5\% patients) followed by diabetic foot 94 (34.9\% patients). When we compared between genders, complication rate was almost equal. A total of 233 patients had poor blood sugar control during their hospitalization and 107 patients had hypertension.

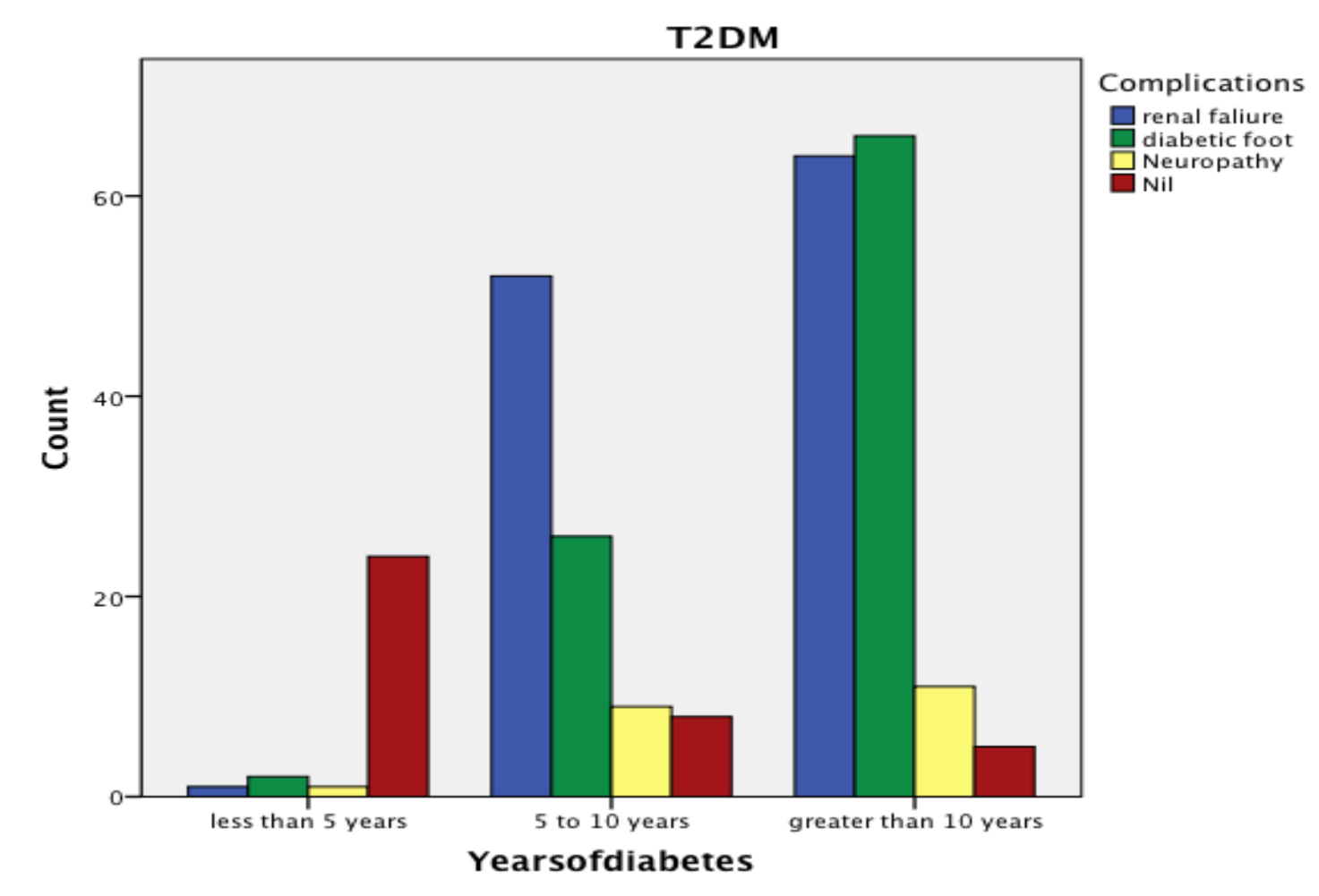

When complication rate was determined over a period of 10 years, the maximum rate was of diabetic foot (66 patients) followed by renal failure (64 patients). The major reason identified was noncompliance to medication and infrequent follow up. Patients with diabetes less than 5 years (24 patients) were without any complication.

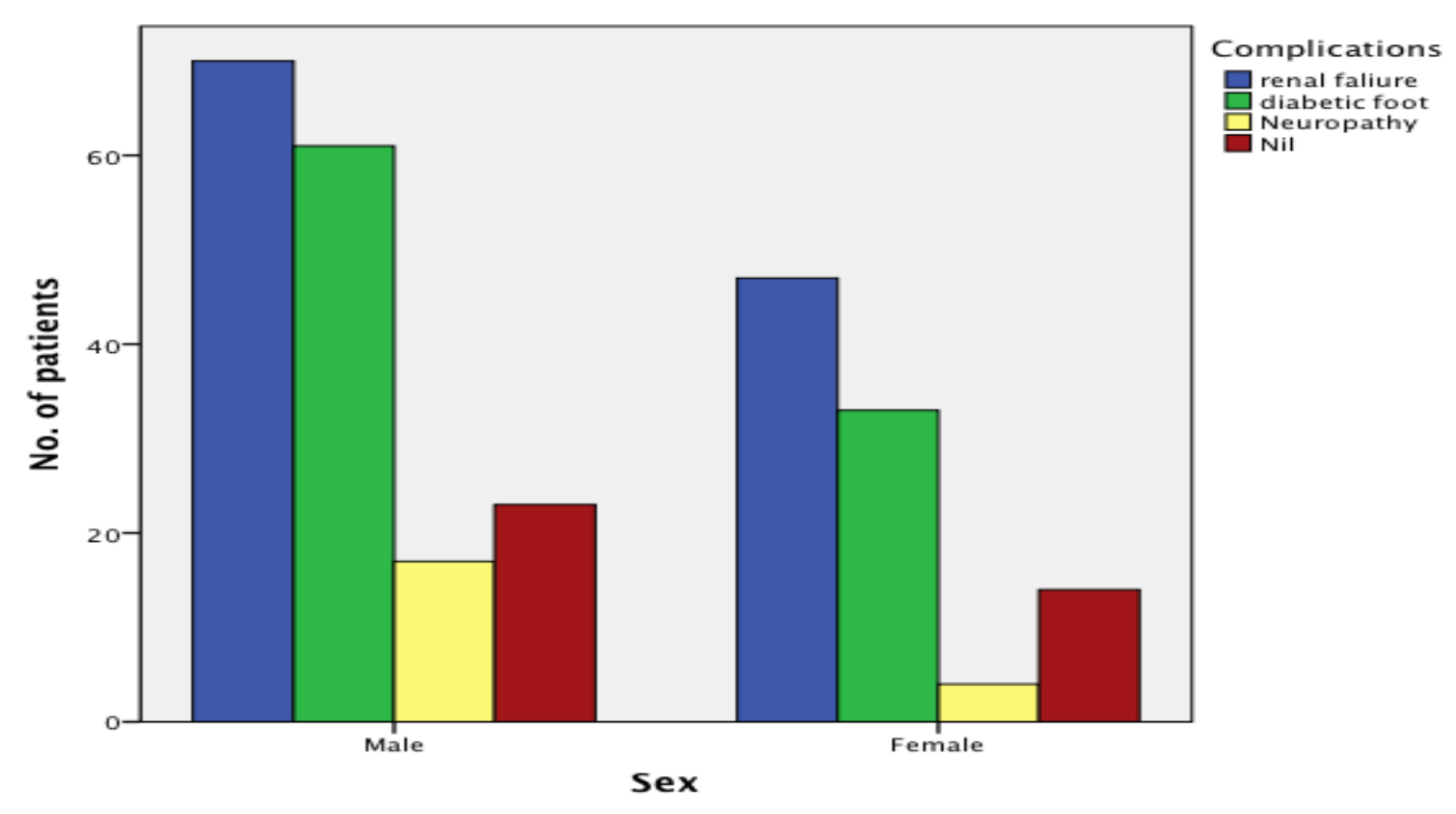

CONCLUSION:

Patients with DM type 2 had a high prevalence of complications. Common diabetic complications among genders were the same. Patients with diabetic complications had high prevalence of risk factors such as poor blood sugar control, concomitant hypertension and poor compliance to medication.
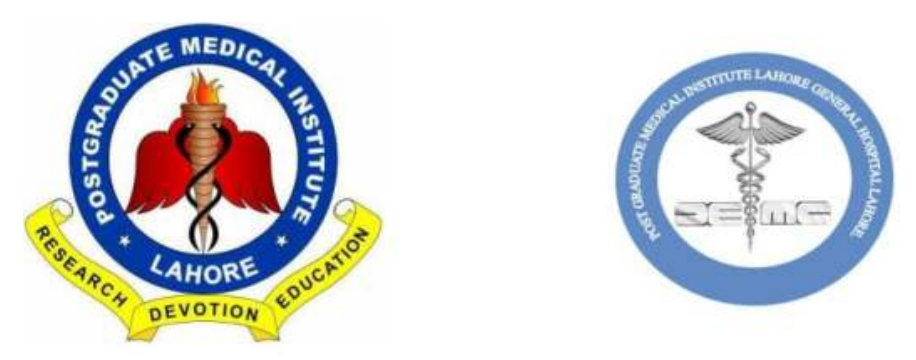